Article

\title{
Open-Source Trading Zones and Boundary Objects: Examining GitHub as a Space for Collaborating on "News"
}

\author{
Mario Haim ${ }^{1, *}$ and Rodrigo Zamith ${ }^{2}$ \\ ${ }^{1}$ Department of Media and Social Sciences, University of Stavanger, 4036 Stavanger, Norway; E-Mail: mario.haim@uis.no \\ 2 Journalism Department, University of Massachusetts Amherst, Amherst, MA 01003, USA; E-Mail: rzamith@umass.edu \\ * Corresponding author
}

Submitted: 30 May 2019 | Accepted: 8 October 2019 | Published: 17 December 2019

\begin{abstract}
New actors, actants, and activities have entered journalism's spaces in recent years. While this has raised the potential for the disruption of existing social orders, such heterogeneous assemblages also provide fruitful grounds for substantive innovation within "trading zones". This article explores one such potential zone, the code-sharing platform GitHub, delineating the primary actors oriented around the boundary object of "news", the objectives of their projects, the nature of their collaborations, and their use of software licenses. The analysis examines attributes of 88,776 news-oriented project repositories, with a smaller subsample subjected to a manual content analysis. Findings show that this trading zone consisted primarily of journalistic outsiders; repositories focused on technological solutions to distributional challenges and efforts that made journalism more transparent; that there was limited direct trade via the use of collaborative affordances on the platform; and that only a minority of repositories employed a permissive license favored by open-source advocates. This leads to a broader conclusion that while GitHub may be discursively important within journalism and certainly provides an avenue for actors to enter journalism's periphery, it offers a limited pathway for those peripheral actors to move closer to the center of journalism. That, in turn, impacts the platform's-and its users'-ability to reconfigure if not spur a reimagining of journalism's meanings, conventions, and allocations of different forms of capital.
\end{abstract}

\section{Keywords}

actors; boundary objects; GitHub; journalism; licenses; news; news innovation; trading zones; transparency

Issue

This article is part of the issue "Peripheral Actors in Journalism: Agents of Change in Journalism Culture and Practice" edited by Avery E. Holton (University of Utah, USA), Valerie Belair-Gagnon (University of Minnesota-Twin Cities, USA), and Oscar Westlund (Oslo Metropolitan University, Norway / Volda University College, Norway / University of Gothenburg, Sweden).

(C) 2019 by the authors; licensee Cogitatio (Lisbon, Portugal). This article is licensed under a Creative Commons Attribution 4.0 International License (CC BY).

\section{Introduction}

Journalism has become a more porous profession than ever before (Lewis \& Zamith, 2017). New actors, actants, and activities have entered its spaces in recent years, raising the potential for the disruption of existing social orders and routines (Lewis \& Westlund, 2015). Scholars have consequently highlighted the growing centrality of technologists and technology companies (Belair-Gagnon \& Holton, 2018; Usher, 2017), the development of algorithms that can perform tasks hitherto restricted to human professionals (Haim \& Graefe, 2017; Wu, Tandoc, \&
Salmon, 2019), and the adoption of new types of labor once seen as external to journalism (Örnebring \& Möller, 2018). Moreover, scholars have sought to explore the interactions among myriad parties interested in journalism, from professional journalists to after-work tinkerers and civic-minded activists (Lewis \& Usher, 2014), some of which aim to 'tweak' journalism while others seek to reimagine it altogether (Lewis \& Usher, 2013).

Such interactions can be particularly meaningful and consequential when they occur within a trading zonea space within which individuals coming from different traditions or with distinct expertise can gather, agree on 
rules of exchange, and engage in complex, coordinated activity around shared goals (Galison, 1997). Within trading zones, participants often engage around boundary objects-concrete or abstract objects that carry different meanings in different social arenas but are sufficiently recognizable as to permit coordination among the members of those distinct realms (Star \& Griesemer, 1989). Such coordination may, in turn, alter meanings, conventions, and the allocation of both symbolic and material resources within specific spaces and a broader field, such as journalism (Lewis \& Zamith, 2017). Notably, the notion of 'news' has been examined as an important boundary object by scholars (e.g., Belair-Gagnon \& Holton, 2018; Boyles, 2019; Lewis \& Usher, 2016).

Drawing on this theoretical lens, the prominent codesharing platform GitHub may serve as an impactful trading zone within which traditional journalistic actors can interact-if not collaborate-with non-traditional actors around the boundary object of news. GitHub is the world's most popular code-sharing and collaboration platform, not least because it is free of charge as long as a project's contents are made public. Individuals-such as technologically proficient journalists, freelance programmers, and data archivists - can create their own projects or add to existing projects developed by others. They may introduce not only code but also documentation and datasets. In short, GitHub represents an interesting site for study as it offers the opportunity for new actors to enter journalistic spaces, be it through collaboration with existing actors or through the introduction of products that are of interest to those spaces (Usher, 2016; Weber \& Kosterich, 2018). However, the existence of technical affordances does not mean they will be leveraged, or leveraged to particular or intended ends (Feldman \& Orlikowski, 2011; see also Boyles, 2017; Wahl-Jorgensen, 2017). That is, just because GitHub offers a platform for trade does not mean trade will occur. This is an empirical question, but one that has not yet been examined by journalism scholars.

This study's examination is carried out by way of a multi-step content analysis that first looks broadly at 88,776 GitHub repositories and then closely evaluates 174 particularly active accounts and 100 of their repositories. This permits an empirical evaluation of the actors that have oriented themselves toward the boundary object of news within a prominent digital space, the extent to which they have collaborated with others, and the goals and outcomes of their endeavors. Indeed, while some researchers have examined journalism vis-à-vis the concepts of trading zones and boundary objects (e.g., Carlson \& Lewis, 2015; Lewis \& Usher, 2016; Smit, de Haan, \& Buijs, 2014), empirical work examining collaborative software development in relation to news is sparse. This leaves important questions unanswered about how journalistic spaces are evolving-from the types of actors that seek to influence them to the nature and impacts of their collaboration to the construction and practice of journalism. This article aims to remedy that by empirically examining how journalism is being reconfigured in a particularly heterogenous and quickly evolving collaborative space. It finds that code-sharing platforms like GitHub provide new spaces for, and involve considerable activity from, actors associated with the periphery of journalism. However, limited trade is presently occurring around the boundary object of news, restricting the ability of journalistic outsiders and their ideas to move from the periphery of journalism to its center. This ultimately points to a missed opportunity for traditional journalistic actors to use code-sharing platforms to work with motivated technological actors in order to develop more innovative actants or more transformative reconfigurations of the field.

\section{Literature Review}

Built upon an ethos of empowerment, today's web allows for extensive collaboration, not least within journalism. A prominent manifestation of those principles is the open-source movement, which leverages code-sharing platforms to promote openness and collaboration on a range of projects (Lewis \& Usher, 2013). As Kelty (2008) argues, open-source is not just an attribute of a project but a philosophy comprised of the following elements: sharing source code, defining openness, writing copyright licenses, coordinating collaborations, and forming a movement. The notion of open-source has not only been applied to journalism but has been heralded as a core component for a fundamental rethinking of journalism (Witt, 2006; see also Baack, 2015).

The most prominent platform dedicated to such collaborative activity is GitHub (Usher, 2016; Weber \& Kosterich, 2018). Such sites are important to study because actors and actants that were not long ago on the periphery of journalism may now be moving closer to the center (Belair-Gagnon \& Holton, 2018), possibly by using collaboration-oriented platforms as their jumping-off points. However, the reconfiguration of journalistic insiders and outsiders (see Eldridge II, 2018) requires participating actors to examine and redefine their own norms and values to suit new orders. A helpful lens for examining whether and the extent to which such potential shifts are taking place may be drawn from the sociological concepts of trading zones and boundary objects, which are synthesized below and applied to the case of GitHub.

\subsection{Trading Zones}

A trading zone refers to the "intermediate domain in which procedures could be coordinated locally even where broader meanings clashed" (Galison, 1997, p. 48). As such, they are spaces within which diverse communities-heterogenous sets of actors, from activists to programmer-journalists, who have their own logics, values, and expertise-come together around shared interests to hammer out solutions to identified problems. That coordination requires the development of suffi- 
cient mutual understanding to permit interdisciplinary productivity. For example, Galison (1997) explored how 20th-century physicists were able to coordinate activities within social spaces and advance the understanding of scientific phenomena despite their distinct cultures, paradigms, and even languages.

As Lewis and Usher (2016, p. 546) argue, trading zones offer "productive possibilities at the intersection of...heterogeneous actors" within the context of journalism. Trading zones allow for the introduction and continuous formation of ideas, perspectives, norms, values, and processes that challenge the status quo (Lewis \& Usher, 2014). These may be consciously embraced or simply adopted through osmosis (Lewis \& Usher, 2016). Notably, some scholars have argued that innovation is more likely to arise from heterogeneous assemblages of competing ideas than homogeneous cooperation (Stark, 2009). Put differently, it is precisely within trading zones that substantive innovation can be expected to occur. Indeed, such spaces may be considered central to the formation of and enaction by "pioneer communities" (Hepp, 2016, p. 924), or collectives that help drive changes in the logics and practices linked to a particular domain (e.g., media). Trade may be consequential to the formation of norms and processes even if it only occurs among some members of distinct communities, so long as those individuals are viewed as opinion leaders within a domain (Zamith, Belair-Gagnon, \& Lewis, 2019).

Scholars have built on the concept of trading zones to highlight the dynamism of such spaces. For example, Collins, Evans, and Gorman (2007) distinguish between different types of trading zones by examining the nature of the collaboration-whether it is cooperative or coerced-and whether the outcome is a heterogeneous or homogeneous culture. They add that a single collaboration can move between different states over time and that a particular trading zone can eventually morph into a new area of expertise. While trading zones may emerge organically, they can also be purposely configured to maximize benefits through the management of constitutional diversity - that is, to limit a priori the kinds of actors who may participate, and the proportion of each form of heterogeneity, in order to reduce unproductive frictions (Sandberg, Holmström, Napier, \& Levén, 2015).

\subsection{Boundary Objects}

Trading zones are typically formed around boundary objects, which refer to "objects which are both plastic enough to adapt to local needs and constraints of the several parties employing them, yet robust enough to maintain a common identity across sites" (Star \& Griesemer, 1989, p. 393). Those objects may be real or imaginary and are interpreted and used in different ways by different communities. However, objects must be rigid enough to be recognizable across more than one domain. Boundary objects serve as core entities that can link communities together and permit heterogeneous sets of actors to col- laborate on a common task within a trading zone. Star and Griesemer's (1989) work, for example, examined how a mix of amateur naturalists, professional scientists, and administrators collaborated to determine which artifacts should be included in the Museum of Vertebrate Zoology at the University of California, Berkeley, and how those objects should then be explained to the museum's visitors.

The notion of 'news' can be one such object (BelairGagnon \& Holton, 2018; Lewis \& Usher, 2016). Although it carries a generally understood outline, it is "clear that journalism is an unstable referent, deployed differently by different actors" (Carlson, 2015, p. 8). As Lewis and Usher (2016) argue, "it may mean certain things to programmers, who have their own occupational and ideological foundations, and something else to journalists" and may thus be considered an object with 'coincident boundaries' under Star and Griesemer's (1989) conceptualization. Such an object is characterized as having common boundaries but different internal contents (Star \& Griesemer, 1989). Put differently, the actors oriented around the boundary object of news may be likely to understand its general contours, but may disagree on the guiding logics for how to best enact the collection, distillation, and distribution of news.

Of particular interest to the intersection of journalism and technology is the value of transparency (Lewis \& Usher, 2013). According to Allen (2008), transparency involves "making public the traditionally private factors that influence the creation of news" (p. 323) and can serve a dual function of improving accountability among news actors and increasing their legitimacy among news audiences. While transparency has long been viewed as an important ideal in journalism, its translation to a commonly enacted ritual has been limited (Singer, 2007). Karlsson (2010) identifies two types of transparency: disclosure transparency and participatory transparency. Disclosure transparency pertains to the degree of openness about how news is selected and produced. This would include making publicly accessible the datasets used in reporting a news story or the code behind a news algorithm. Participatory transparency pertains to the extent to which audiences are incorporated into the selection and production of news. This would include inviting audiences to contribute to ongoing newswork, such as by adding functionality to a news product or merging new data into existing datasets. Scholars have argued that the affordances of digital journalism provide opportunities for transparency that are not possible with its analog counterparts (Karlsson \& Holt, 2016), with some suggesting transparency to now be one of journalism's most important values (Vos \& Craft, 2017).

\subsection{Sociotechnical Collaboration in Journalism}

In the theoretical realm of trading zones and boundary objects, the scope of journalism and its set of relevant actors is fluid, inviting new actors to enter spaces and 
help reshape them (Lewis \& Zamith, 2017). However, as scholars have argued, a shared sense of journalistic professionalism-and of belonging to the core spaces of 'journalism' -is central to insiders' claims of authority and legitimacy within the realm of news production, and to the acceptance of such claims by journalistic outsiders (Carlson, 2017). Transparency, both in its disclosure and participatory forms, provides fruitful grounds for engagement among a variety of actors by introducing pathways for collaboration and demystifying boundary objects. Over time, that collaboration strengthens the meanings associated with a boundary object (e.g., news) among the members of a given trading zone and may subsequently discursively reconstitute those objects within a broader domain (e.g., journalism). Put differently, collaboration is important not only because of its material output but because it can simultaneously reify and reshape meanings across spaces (Baack, 2015; Lewis \& Usher, 2016).

The availability of collaboration affordances need not mean they will be used, though (Feldman \& Orlikowski, 2011). Indeed, journalism is filled with failed sociotechnical experiments designed to invite collaboration among and across journalistic and non-journalistic actors (WahlJorgensen, 2017). It is similarly filled with actants that never gained acceptance or stopped being developed and maintained (Boyles, 2017). The notion of sustainability has thus received attention by scholars of innovation, who have highlighted the importance of active communities and collaboration to a project's long-term success (Lewis \& Usher, 2014). Heikka and Carayannis (2019) argue that digital spaces alone are exclusionary of individuals lacking technological ability, and that "collaboration may not cross the boundaries that need to be crossed" (p. 458) to generate substantive and meaningful innovation. Important participation gaps have also been found in open-source and code-sharing spaces, with women and minorities being far less likely to engage or contribute (Ensmenger, 2015; Ford, Smith, Guo, \& Parnin, 2016). Instead, Heikka and Carayannis (2019, p. 440) point to the importance of creating offline "holding spaces" where multiple community stakeholders can co-initiate, co-sense, and co-create different innovations. Not all collaboration is useful, though. Indeed, some trading zones may prove unproductive as more time is spent negotiating meanings and frictions than advancing ideas (Smit et al., 2014).

There are myriad motivations for participating in open-source projects, from the desire to work on challenging problems to wanting to participate in something big (Weber, 2004). Few contributors participate primarily to make money (Jordan, 2017), but many do seek symbolic reward in the form of status within an interpretive community (Marlow, Dabbish, \& Herbsleb, 2013). Individuals associated with prominent projects or organizations, high quantities of contributed code, and long-standing user profiles are often granted higher status, and that reputational capital can be translated into greater acceptance and impact for their creations as well as an increased likelihood of successfully soliciting contributions from others (Dabbish, Stuart, Tsay, \& Herbsleb, 2013). However, it is important to note that while some members may orient themselves toward the space of professional journalism, they may not see themselves as journalists. As Baack (2015) argues, many act as "intermediaries outside the profession" (p. 6), with some aiming to advance journalistic ideas and ideals through the creation of independent, civic technologies, and others seeking to incentivize news organizations to imitate open-source projects. As one interviewee told Baack (2015), "we have discovered software as a lobbying tool" (p. 7) to get institutional actors like news organizations to adopt their ideas, values, and priorities. Within newsrooms, participation in open-source communities is often an attempt to expand and find belonging in a broader interpretive community and to gain status by claiming particular contributions of code (Boyles, 2019). In doing so, newsroom developers help to institutionalize journalistic norms and ideals within open-source spaces while translating those of open-source spaces across the news organization (Usher, 2016).

Despite the theoretical utility of the concepts of trading zones and boundary objects, their empirical application to the study of sociotechnical collaboration within journalism has been limited. Lewis and Usher (2014) used it to find that the lack of sustained and enduring exchanges made it difficult for chapters of Hacks/Hackers - a transnational grassroots organization that bridges journalists and technologists-to generate momentum for more in-depth sharing, especially when there was limited support from local institutions. Lewis and Usher (2016) analyzed participation in a news innovation-oriented 'learning lab' and found that distinct understandings of news and technology converged, diverged, and ultimately blended around the themes of making news more process-oriented, participatory, and socially curated. Smit and colleagues (2014) examined information-visualization production at three different organizations and identified four different types of cooperation that can be adapted to suit different kinds of trading zones. Moreover, throughout the broader stream of work examining the intersection of journalism and technology, scholars have found a growing desire among journalists to collaborate with technologically oriented actors in order to develop technologically infused products and processes that advance journalistic values (see Lindén, 2017). Boyles (2019), for example, found that "newsroom developers uniformly stated that participation within OSS (open-source) environments is a core responsibility of their positions" (p. 10).

\subsection{GitHub and Software Licensing}

Within the context of software, and the broader intersection of journalism and technology, GitHub is a central site for interdisciplinary activity (Tsay, Dabbish, \& 
Herbsleb, 2014). GitHub is a web-based hosting service that builds on the popular Git version-control software, allowing incremental updates to digital files to be logged, publicly distributed, and collaboratively worked upon. Projects on GitHub are organized around 'repositories,' which are akin to folders containing source code and raw data made available for others to review, improve, and collaborate around. Projects, which may include software like web scrapers as well as documents like Python Notebooks detailing a data-journalistic analysis, are typically oriented around a single repository, though they may theoretically be broken up into multiple repositories. Each repository is labeled with a title or name and, optionally, a description of the code's purpose. Repository owners can add regular maintainers, merge ad-hoc contributions, and have their repositories 'forked' to be built upon independently by others. GitHub also provides mechanisms for repository-specific bug tracking, feature requests, task management, and wikis, thereby welcoming contributions from non-programmers as well. Users can utilize the aforementioned features, including the creation and accessing of repositories, for free. As of late 2019, GitHub had more than 37 million users and an equivalent number of public repositories, making it the world's largest code repository.

The platform is also used by a range of news organizations (e.g., The New York Times and BuzzFeed News) to share datasets, data-analysis methods, and newsroom innovations as well as to invite audience participation. Boyles (2019) found that several newsrooms that had received a data-journalism award had organizational GitHub accounts or had newsroom developers who had an account. Although the accounts shared ample contact information and offered affordances for engagement, Boyles observed limited interaction with other users - and little external activity involving project files. This led Boyles to conclude that GitHub primarily served as a signaling platform: It helped to brand the organization's prestige in open-source spaces, conveying a commitment to openness and reciprocity while increasing the visibility and reputation of its own contributions to that space.

GitHub also provides easy-to-use mechanisms for licensing software and promotes their use. When setting up a repository-and at any time during the development process-users can compare different licensing models and easily apply them to their repositories. Applied licenses appear prominently inside the repositories and signal the degrees of permission or prohibition for how others may use, extend, modify, or redistribute project files (Vendome et al., 2015). For example, some licenses permit the unrestricted use (i.e., commercial or personal) of the repository's contents and derivatives, while others require derivatives to use the same license. The selection of a license may have bearing on the amount and kind of trade and collaboration that takes place around a repository as licenses are a legally enforceable expression of a repository owner's in- tent (Almeida, Murphy, Wilson, \& Hoye, 2019). Put differently, permissive licenses may encourage collaboration and promote sustainability while restrictive licenses may promote greater control by the repository owner, which may limit such possibilities.

\subsection{Research Questions}

The literature shows that journalism has become an increasingly porous field, with nontraditional actors becoming more central to its functioning (Belair-Gagnon \& Holton, 2018; Lewis \& Zamith, 2017; Örnebring \& Möller, 2018). This opens up opportunities for trade around the boundary object of 'news,' which can reshape and reify key material objects, normative processes, and professional discourses (Carlson, 2015; Lewis \& Usher, 2016; Weber \& Kosterich, 2018). Those efforts are most fruitful when the trade cohort is heterogeneous and active, though there are multiple structural obstacles to the success of trade and the sustainability of its outputs (Lindén, 2017; Smit et al., 2014; Weber \& Kosterich, 2018). In light of this work, the following research questions are posed:

RQ1: Who are the primary actors gathering around the object of news on GitHub?

$\mathrm{RQ2:}$ What are the objectives of news repositories on GitHub?

RQ3: How much collaboration exists within news repositories on GitHub across different development affordances?

RQ4: What are the software licenses used by news repositories on GitHub?

\section{Method}

\subsection{Data Collection}

The GitHub application programming interface (API) was used to capture a wide range of data for the population of news-related repositories, which effectively serve as folders for storing project files, created on GitHub. This included all repositories in which the terms 'news' or 'journalism' appeared in the name or description fields. Since GitHub's API restricts the number of results per request, several requests were formulated to ensure data were collected for all relevant repositories. Data collection took place on September 11, 2018 and yielded a total of 88,776 repositories (i.e., projects), with the vast majority $(98.3 \%)$ containing the term 'news.'

\subsection{Coding Procedure}

The study adopted a three-step procedure to make possible both a broad analysis of all repositories and a close analysis of the most influential actors and repositories. In the first step, the 'amount of collaboration' was evaluated by reviewing API-derived information for all repositories, including the number of forks (direct derivations 
of a repository), stars (bookmarks to that repository), and time lapse between the repository creation and its last update, as well as the number of contributors for some of the repositories (manually coded as described below). The 'software licenses' were also evaluated by using that API information as GitHub collects this information based on either an author's self-report or their inclusion of a license file within their repository.

In the second step, all user accounts with more than five associated repositories in the data collection were sampled. This was done to remove noise from the datathat is, less consequential actors whose contributions were likely relatively minor. This step resulted in 174 unique actors, accounting for a total of 2,447 repositories. Those actors were then manually coded in a manner consistent with the approach by Dabbish, Stuart, Tsay, and Herbsleb (2012). Specifically, an inductive categorygeneration process was used to establish the following categories for 'actor type': digitally native news organization, educational organization, interest group, legacy news organization, private organization, public organization, unaffiliated individual, and not identifiable or other. While 'unaffiliated' and 'not identifiable' actors may seem conceptually similar, they are distinct in that the former choose to divulge identifying information but not leverage a connection to any organization whereas the latter choose or fail to provide minimal information overall. Then, an account's username, optional biography and external link, associated repositories, and any clearly observable suggestions of the owner's identity were evaluated to deductively code each account. All accounts were double-coded by the authors and any disagreements discussed and resolved through a process of expert coding (see also Hermida, Lewis, \& Zamith, 2014).

In the third step, a stratified random sample of 100 repositories was drawn from those 2,447 repositories of the most-active users. Each repository was again doublecoded using the aforementioned process. First, the following categories for 'project objectives' were generated inductively: news production materials (e.g., supplemental data for published articles), means for news consumption (e.g., offline reader apps), means for news distribution (e.g., website plugin code), means for news production (e.g., newsletter builder program), information access (e.g., tools for facilitating API access), information literacy (e.g., educational materials), and non-news projects or those not readily identifiable. Then, a deductive coding process evaluated the repository's name, optional description and external link, and any materials available in the repository, such as 'ReadMe' files or raw source code.

All data and analyses are publicly available on the Harvard Dataverse, under https://doi.org/10.7910/DVN/ LUOZJL.

\section{Results}

\subsection{Primary Actors}

The set of actors gathering around the object of news followed a long-tail distribution, as the majority of user accounts in the dataset (89.2\%) created just one newsrelated repository. Just 174 account-holders matched the five-repository sampling criterion - the distribution's head, which is deemed to be of particular importance given their outsized contribution of 2,447 repositories (see Table 1).

Among the sampled accounts, unaffiliated individuals $(20.1 \%$, or $n=35)$ accounted for the highest number of accounts, followed by educational organizations $(15.5 \%, n=27)$, private organizations $(15.5 \%, n=27)$, interest groups $(12.1 \%, n=21)$, legacy news organizations $(9.8 \%, n=17)$, digitally native news organizations $(5.2 \%$, $n=9)$, and public organizations $(1.7 \%, n=3)$. There was a non-negligible number of accounts $(20.1 \%, n=35)$ that could not be clearly associated with an actor type due to lack of information. This may yield an under-counting of some actor types that are more likely to display less information, including unaffiliated individuals.

Additionally, certain groups were more prolific in producing repositories than others. Accounts associated with interest groups produced an exceptionally large share of repositories $(32.8 \%$, or $n=802)$ given its proportion of account-holders. They were followed by unaffiliated individuals $(11.0 \%, n=269)$, who can be understood as human beings willingly presenting themselves as not being attached to any organization. The remaining groups include private organizations $(10.0 \%, n=244)$,

Table 1. Actors on GitHub based on an analysis of a subset of news-related accounts and repositories.

\begin{tabular}{llcc}
\hline Actor Type & Example & $\boldsymbol{n}$ of Actors $(\boldsymbol{N}=\mathbf{1 7 4})$ & $\boldsymbol{n}$ of Repositories $(\boldsymbol{N}=\mathbf{2 , 4 4 7})$ \\
\hline Digital Native News Organization & BuzzFeedNews & 9 & 208 \\
Educational Organization & TowCenter & 27 & 238 \\
Interest Groups & OpenNewsLabs & 21 & 802 \\
Legacy News Organization & SeattleTimes & 17 & 230 \\
Private Organization & IBM & 27 & 244 \\
Public Organization & bcgov & 3 & 54 \\
Unaffiliated Individual & jonathanheilmann & 35 & 269 \\
Not Identifiable & hamsmo & 35 & 402 \\
\hline
\end{tabular}

Note: Only actors (i.e., user accounts) with more than five repositories are included. 
educational organizations $(9.7 \%, n=238)$, legacy news outlets $(9.4 \%, n=230)$, digitally native news organizations $(8.5 \%, n=208)$, and public organizations $(2.2 \%$, $n=54)$. Accounts that could not be clearly associated with an actor type accounted for $16.4 \%(n=402)$ of the repositories.

\subsection{Primary Objectives}

Of the 100 manually coded repositories (i.e., projects), 28 focused on means for news distribution, such as WordPress plugins for organizing recent headlines. This was followed by news production materials like copies of datasets $(n=15)$, information literacy resources like tutorials ( $n=12$ ), information access tools like automated web scrapers $(n=10)$, means for news production like newsletter builders $(n=6)$, and means for news consumption like RSS (Really Simple Syndication) readers $(n=3)$. Additionally, three repositories were not directly related to news, and another 23 could not be clearly identified due to language barriers or lack of information.

\subsection{Collaboration and Development}

The majority (73.6\%) of all 88,776 repositories contained multiple 'commits,' or incremental contributions. Additionally, the projects' median lifetime, calculated as the time elapsed between a repository's creation and its latest update, was 17 weeks. As such, news-related repositories did provide opportunities for collaboration and trade since the window for such engagement was open for almost four months on average, at which point the project either reached maturation or was abandoned.

Despite those opportunities, collaboration on GitHub was the exception rather than the norm. The vast majority of repositories $(89.7 \%)$ among all 88,776 repositories were never 'forked' by other users for further development. Just $6.0 \%$ were forked once, $2.8 \%$ two to five times, and $1.5 \%$ six times or more. Similar results were found for the starring mechanism. The vast majority of repositories $(84.5 \%)$ were never starred. Only $8.2 \%$ received one star, $4.5 \%$ were starred two to five times, and $2.8 \%$ were starred six or more times.

Those results were generally consistent among the subsampled repositories. Among the 100 manually coded repositories, 83 were never forked, 10 were forked once, 3 were forked two to five times, and 4 were forked more than six times. Similarly, 82 were never starred, 11 were starred once, 6 were starred two to five times, and just one was starred more than six times. Moreover, 82 of the repositories only received contributions from the repository owner, with 12 receiving contributions from two people, and 6 from three or more contributors.

\subsection{Licenses Used}

While GitHub encourages users to specify a license when they create a repository, there is no requirement to do so. As a result, only $15.2 \%$ of the 88,776 repositories included a license. Among the licensed projects, the majority employed a derivative of the MIT License (51.4\%), GNU General Public License (17.9\%), or Apache License (14.2\%). Seventeen other licenses accounted for $16.5 \%$ of the repositories.

Turning to the sample of repositories from the manually coded actors ( $n=2,447)$, slightly higher rates of license inclusion become apparent. That is, $22.0 \%$ of those projects employed a license, with an almost equal distribution across the major license types of MIT (26.2\%), GNU (22.6\%), and Apache (25.4\%) derivatives. This does not come as a surprise as the sampling for the 2,447 repositories emphasized heavy users (i.e., those who had more than five repositories in the sampling frame). Those individuals likely have more experience with opensource software.

Breaking down the licenses used by actor types among that manually coded subset, $57.4 \%$ of the repositories from public organizations and $39.8 \%$ of the repositories from private organizations employed a license. Interestingly, almost half (49.1\%) of legacy news organizations used a license, the majority of which (66.4\%) drew on the Apache model, a relatively restrictive license. Similarly, public organizations mainly employed Apache licenses (80.6\%). In contrast, private organizations primarily used GNU derivatives (47.4\%), a 'strong copyleft' license.

\section{Discussion}

This study provides empirical evidence for evaluating previously raised assumptions about the growing interplay of actors and actants from both inside and outside journalism around the boundary object of 'news.' The GitHub trading zone consisted primarily of journalistic outsiders who aimed to offer technological solutions to distributional challenges and to make journalism more transparent. However, despite its affordances and intent, the zone exhibited little direct trade among actors and introduced structural barriers to trading.

That the space for journalism on GitHub was made up primarily of different journalistic outsiders introduces opportunities for the clash of distinct logics and meanings (Galison, 1997). It also offers further empirical support for scholars' contentions that actors once seen as being outside of journalism are increasingly entering, or at least trying to enter, its spaces (Belair-Gagnon \& Holton, 2018; Eldridge II, 2018). That many of the contributors analyzed were unaffiliated individuals highlights the growing number of 'tinkerers' drawn to journalism (see Lewis \& Usher, 2014) and raises important questions about the perceived legitimacy and authority of peripheral actors lacking institutional backing (Carlson, 2017), and how social capital is redistributed within highly dynamic spaces (Lewis \& Zamith, 2017). It is plausible that such individuals, lacking the social capital accorded to actors associated with recognizable affiliates 
(e.g., Facebook or The New York Times), may see GitHub as a more merit-oriented platform wherein contributions are evaluated in terms of technological worth (e.g., wellwritten code) rather than the contributor's attributesthough it should be noted that certain groups face considerable non-merit barriers (Ensmenger, 2015; Ford et al., 2016; Heikka \& Carayannis, 2019). From this vantage point, GitHub may be seen as an accessible pathway for entering and influencing journalism by actors outside journalism's immediate orbit, helping to explain the participation by traditionally peripheral actors (see also Baack, 2015). Moreover, the fact that one-fifth of the coded accounts were not identifiable at all could also indicate that these findings might be underestimated. It is important to note, however, that unidentifiable actors simply failed to provide sufficient information to enable other users to evaluate their identity whereas unaffiliated individuals provided a clear sense of identity but chose not to connect themselves with any particular organization. They are, therefore, distinct groups. Furthermore, that the vast majority of actors were not connected to a news organization underscores that news is very much a boundary object (Belair-Gagnon \& Holton, 2018; Lewis \& Usher, 2016) and that journalism continues to be seen as an interesting context to which technically minded individuals may apply their skills (Usher, 2016). However, the limited involvement by individuals working at news organizations may also be viewed as evidence of those organizations' limited embrace of outsiders and their ideas, at least on code-sharing platforms such as GitHub (cf. Boyles, 2019). It also raises questions about the authoritative control that news organizations have on these platforms. For example, might this moreheterogenous set of actors potentially be coopting the language of journalism and redefining what 'news' and 'journalism' might mean outside of professional journalistic spaces?

Strikingly, the amount of software from journalistic outsiders designed for news distribution underscores the growing emphasis on distribution within and beyond journalism (Ferrer-Conill \& Tandoc, 2018; Zamith \& Braun, 2019). In combination with the findings suggesting GitHub to be a platform where technological solutions are promoted primarily by technologists, this illustrates a shift noted in the literature toward a more technology-centered view of journalism, where actants are used to make news more accessible (Lindén, 2017; Zamith, 2019).

The findings also point to the use of the platform to advance both disclosure and, to a lesser extent, participatory forms of transparency (Karlsson, 2010). This was evident in the use of the platform to share data used in journalistic endeavors - the primary use case for repositories associated with news organizations. It was also used as a platform to educate those interested in (data-driven and computational) journalism. Such projects support scholars' contention that journalism is increasingly prioritizing transparency (Karlsson \& Holt, 2016; Vos \& Craft,
2017) and translating it into an enacted ritual (cf. Singer, 2007). This may also be viewed as evidence that the openness aspect of the open-source ethos may be permeating journalism culture as certain outsiders enter its spaces (Baack, 2015; Lewis \& Usher, 2013, 2016). Put differently, while disclosure transparency has long been a feature of journalism's ideals (Singer, 2007), the manner in which it is put into practice today leverages technologies typically viewed as being on the periphery of journalistic spaces, such as code-sharing platforms (see also Boyles, 2019).

However, the findings also suggest that limited trade occurs on GitHub when it comes to news-related projects. This was evidenced by presence of multiple commits that, on average, would span nearly four months. However, project repositories rarely had more than a single contributor (the repository owner)perhaps the most salient marker of direct trade. Moreover, they were rarely 'forked,' which could be viewed as a measure of independent development influenced by a trade partner, or 'starred' by others. Consequently, GitHub may be viewed as a cooperative trading zone in light of its voluntary and ad-hoc nature (see Collins et al., 2007), but for news it is perhaps best described as an inactive trading zone. It may nevertheless remain important as a discursive object, as evidenced by Usher's (2016) finding that GitHub itself served as a discursive nexus around which members of hackathons can be oriented (see also Weber \& Kosterich, 2018). It may also serve a valuable signaling function wherein organizations can convey their commitments to certain ideals, and through which individuals-and insiders like newsroom developers in particular-can seek to gain reputational capital (Boyles, 2019). However, even if the amount of trade is limited, it may nevertheless prove influential if the individuals doing the trade are viewed as opinion leaders within their respective domains (see Zamith et al., 2019).

That key finding underscores the challenge of uniting disparate groups around a boundary object (Smit et al., 2014; Star \& Griesemer, 1989). For example, Lewis and Usher (2014) found that limited organizational support and a lack of sustained and enduring exchanges made it hard for Hacks/Hackers groups to maintain momentum. Similarly, Heikka and Carayannis (2019) argued that a broader infrastructure comprised of institutional actors and civic-minded individuals was central to creating formal and informal spaces for promoting journalistic innovation. The lack of trade around projects and limited institutional activity might similarly impair their sustainability, further adding to the record of failed sociotechnical interventions (Boyles, 2017; Wahl-Jorgensen, 2017). Importantly, innovation is most likely to arise from heterogeneous assemblages (Stark, 2009) and be most impactful when a community spans disciplines (Galison, 1997). From that perspective, GitHub and the news projects within it may be neither particularly innovative nor especially impactful, thus limiting their ability to re- 
shape the boundaries of journalism (see Carlson \& Lewis, 2015) and its associated meanings, conventions, and activities (Lewis \& Westlund, 2015; Lewis \& Zamith, 2017). This also presumably limits its ability to serve as a cornerstone for the formation of news-oriented "pioneer communities" (Hepp, 2016, p. 924) that can be catalysts for deeper changes. Transformative change is thus more likely to succeed if it is rooted in planned social activity that is designed to promote cross-actor dialoguerather than expecting dialogue to naturally emerge because the technological affordances to support it exist (Heikka \& Carayannis, 2018). While such change need not be led by news organizations, their engagement with external actors can inform innovations within newsrooms and help maintain their relevance and legitimacy as civically oriented actors (Baack, 2015; Heikka \& Carayannis, 2018). Ultimately, it appears that while GitHub may be perceived by peripheral actors as an accessible pathway to influence journalistic spaces-should that be their objective-the evidence suggests it is unlikely that they are gaining authority and legitimacy among journalistic insiders as a result of those efforts, or significantly influencing insiders' conceptions through the exchange of expertise or products.

The limited collaboration and impact may also be due to very few repositories employing a license. This observation contrasts previous non-news findings indicating that software developers are well-aware of the necessity of license use (Almeida et al., 2019). While the initial act of publicly listing a project on GitHub may serve as an invitation for collaboration, building on non-licensed projects is a legally gray area (see Vendome et al., 2015). Indeed, the overwhelming majority of repositories may not be legally 'forkable' or able to be included as a subcomponent of a larger project. Beyond the legal implications, licenses are signals for inviting and even encouraging collaboration. When used by news repositories, licenses placed relatively few restrictions (e.g., MIT, GPL). While the limited amount of licensing is unlikely to be a primary cause of the low levels of collaboration, it is something that actors can easily resolve.

It is important to recognize that this study did not evaluate reported issues or wikis-affordances on GitHub that permit ideas to be more easily exchanged by non-technical actors. Some trade may be occurring through those mechanisms, even if those ideas have not yet been translated to code. Additionally, the study only evaluated one code-sharing platform; important competitors like GitLab and SourceForge were not evaluated. The close analysis also only looked at a small subset of accounts, and omitted an alternative approach to evaluating impact that might have focused on repositories with a large number of commits and/or forks.

Moreover, this study did not distinguish between 'news' and 'journalism' repositories, but instead treated them as a singular concept. These terms may carry distinct connotations to certain actors and be used intentionally in positioning their projects (see Harcup, 2014).
For example, certain interest groups may seek to develop tools that facilitate the dissemination of 'news' pertaining to their activities, without purporting that information to be 'journalism.' Within the context of this analysis, it was found that the vast majority of repositories were associated with the term 'news' and the authors found evidence of the terms being used interchangeably even by traditional journalism organizations (e.g., The Guardian and the Los Angeles Times), which one would expect to be most sensitive to the terminology. This suggests that these two terms, which are conceptually distinct in the literature, may not be viewed so distinctly in professional practice (and among non-professionals). A post-hoc analysis to empirically evaluate differences within the collected data indicated that interest groups and private organizations were more likely to produce 'news'-related projects than 'journalism'-related projects, while educational organizations and legacy news outlets were more likely to produce 'journalism'-related projects. However, the small scale of that post-hoc analysis precludes the presentation of generalizable findings, especially with regard to the objectives of projects that used particular terminology. As such, a closer examination of the linguistic patterns of such projects and how they implicate their positioning and objectives would be fruitful to the scholarly understanding of how such terms are conceptualized by a broad, technologically oriented set of actors.

Future work may also opt to focus on forums used to exchange technical knowledge and values, like StackOverflow and Google Groups. Those venues are often helpful starting points for non-technological individuals and frequently patronized by their technologically oriented counterparts. Similarly, interviewing actors who use GitHub might yield insights into their motivations and the barriers they face in trading, allowing scholars to examine how structural barriers found in other domains manifest in the realm of journalism (see Ensmenger, 2015; Ford et al., 2016; Heikka \& Carayannis, 2019). Finally, the existing work on Hacks/Hackers (e.g., Lewis \& Usher, 2014) can be extended to examine their (non-)use of GitHub. Indeed, purposively sampling those technologically minded individuals already drawn to the idea of collaboration may shed light into why some choose not to participate in news-related projects on code-sharing platforms (see also Boyles, 2019). It would also behoove future research to examine offline and online spaces simultaneously, rather than in isolation (see Heikka \& Carayannis, 2018).

In conclusion, code-sharing platforms like GitHub provide spaces for a heterogeneous set of actors to congregate around the boundary object of 'news,' with the analysis offering further empirical evidence that new actors are seeking to break into journalistic spaces. However, the small amount of trade activity suggests the platform has a limited ability to serve as a conduit for transporting journalistic outsiders from the periphery of journalism to its center. It further points to a narrow em- 
brace of the open-source ethos, though vestiges may be found in the growing focus on transparency facilitated by GitHub. Ultimately, there appears to be a missed opportunity for newsrooms to use code-sharing platforms to integrate more closely with motivated technological actors-and potentially engage in the development of more innovative actants or more transformative reconfigurations of the field.

\section{Acknowledgments}

The authors would like to thank the editors of the thematic issue, the two anonymous reviewers, and the participants of the inaugural symposium on Media, Professions and Society in Volda, Norway, for their thoughtful feedback throughout the process of writing this article.

\section{Conflict of Interests}

The authors declare no conflict of interests.

\section{References}

Allen, D. S. (2008). The trouble with transparency: The challenge of doing journalism ethics in a surveillance society. Journalism Studies, 9(3), 323-340. https:// doi.org/10.1080/14616700801997224

Almeida, D. A., Murphy, G. C., Wilson, G., \& Hoye, M. (2019). Investigating whether and how software developers understand open source software licensing. Empirical Software Engineer, 24(1), 211-239. https:// doi.org/10.1007/s10664-018-9614-9

Baack, S. (2015). Datafication and empowerment: How the open data movement re-articulates notions of democracy, participation, and journalism. Big Data \& Society, 2(2), 1-11. https://doi.org/10.1177/ 2053951715594634

Belair-Gagnon, V., \& Holton, A. E. (2018). Boundary work, interloper media, and analytics in newsrooms. Digital Journalism, 6(4), 492-508. https://doi.org/10.1080/ 21670811.2018.1445001

Boyles, J. L. (2017). Laboratories for news? Experimenting with journalism hackathons. Journalism. Advance online publication. https://doi.org/10.1177/ 1464884917737213

Boyles, J. L. (2019). Deciphering code: How newsroom developers communicate journalistic labor. Journalism Studies. Advance online publication. https://doi.org/ 10.1080/1461670X.2019.1653218

Carlson, M. (2015). The many boundaries of journalism. In M. Carlson \& S. C. Lewis (Eds.), Boundaries of journalism: Professionalism, practices and participation (pp. 1-18). London: Routledge.

Carlson, M. (2017). Journalistic authority: Legitimating news in the digital era. New York, NY: Columbia University Press.

Carlson, M., \& Lewis, S. C. (2015). Boundaries of jour- nalism: Professionalism, practices and participation. New York, NY: Routledge.

Collins, H., Evans, R., \& Gorman, M. (2007). Trading zones and interactional expertise. Studies in History and Philosophy of Modern Physics, 38(4), 657-666. https://doi.org/10.1016/j.shpsa.2007.09.003

Dabbish, L., Stuart, C., Tsay, J., \& Herbsleb, J. (2012). Social coding in GitHub: Transparency and collaboration in an open software repository. In Proceedings of the ACM 2012 conference on computer supported cooperative work (pp. 1277-1286). New York, NY: ACM. https://doi.org/10.1145/2145204.2145396

Dabbish, L., Stuart, C., Tsay, J., \& Herbsleb, J. (2013). Leveraging transparency. IEEE Software, 30(1), 37-43. https://doi.org/10.1109/MS.2012.172

Eldridge II, S. A. (2018). Online journalism from the periphery. London: Routledge.

Ensmenger, N. (2015). "Beards, sandals, and other signs of rugged individualism": Masculine culture within the computing professions. Osiris, 30(1), 38-65. https://doi.org/10.1086/682955

Feldman, M. S., \& Orlikowski, W. J. (2011). Theorizing practice and practicing theory. Organization Science, 22(5), 1240-1253. https://doi.org/10.1287/ orsc. 1100.0612

Ferrer-Conill, R., \& Tandoc, E. C. (2018). The audienceoriented editor. Digital Journalism, 6(4), 436-453. https://doi.org/10.1080/21670811.2018.1440972

Ford, D., Smith, J., Guo, P. J., \& Parnin, C. (2016). Paradise unplugged: Identifying barriers for female participation on stack overflow. In Proceedings of the 24th international symposium on foundations of software engineering (pp. 846-857). New York, NY: ACM. https://doi.org/10.1145/2950290.2950331

Galison, P. (1997). Image and logic: A material culture of microphysics. Chicago, IL: The University of Chicago Press.

Haim, M., \& Graefe, A. (2017). Automated news. Digital Journalism, 5(8), 1044-1059. https://doi.org/10. 1080/21670811.2017.1345643

Harcup, T. (2014). A dictionary of journalism. Oxford: Oxford University Press.

Heikka, T., \& Carayannis, E. G. (2018). The role of journalism in dialogic innovation processes: The case of the Helsinki deaconess institute multi-stakeholder workshops. Journal of the Knowledge Economy, 9(4), 1415-1441. https://doi.org/10.1007/s13132016-0427-z

Heikka, T., \& Carayannis, E. G. (2019). Three stages of innovation in participatory journalism: Co-initiating, co-sensing, and co-creating news in the Chicago school cuts case. Journal of the Knowledge Economy, 10(2), 437-464. https://doi.org/10.1007/ s13132-017-0466-0

Hepp, A. (2016). Pioneer communities: Collective actors in deep mediatisation. Media Culture \& Society, 38(6), 918-933. https://doi.org/10.1177/ 0163443716664484 
Hermida, A., Lewis, S. C., \& Zamith, R. (2014). Sourcing the Arab spring: A case study of Andy Carvin's sources during the Tunisian and Egyptian revolutions. Journal of Computer-Mediated Communication, 19(3), 479-499.

Jordan, T. (2017). A genealogy of hacking. Convergence, 23(5), 528-544. https://doi.org/10.1177/ 1354856516640710

Karlsson, M. (2010). Rituals of transparency. Journalism Studies, 11(4), 535-545. https://doi.org/10.1080/ 14616701003638400

Karlsson, M., \& Holt, K. (2016). Journalism on the web. In J. F. Nussbaum (Ed.), Oxford research encyclopedia of communication. Oxford: Oxford University Press.

Kelty, C. M. (2008). Two bits: The cultural significance of free software. Durham, NC: Duke University Press.

Lewis, S. C., \& Usher, N. (2013). Open source and journalism: Toward new frameworks for imagining news innovation. Media Culture \& Society, 35(5), 602-619. https://doi.org/10.1177/0163443713485494

Lewis, S. C., \& Usher, N. (2014). Code, collaboration, and the future of journalism. Digital Journalism, 2(3), 383-393. https://doi.org/10.1080/21670811. 2014.895504

Lewis, S. C., \& Usher, N. (2016). Trading zones, boundary objects, and the pursuit of news innovation: A case study of journalists and programmers. Convergence, 22(5), 543-560. https://doi.org/10.1177/ 1354856515623865

Lewis, S. C., \& Westlund, O. (2015). Actors, actants, audiences, and activities in cross-media news work. Digital Journalism, 3(1), 19-37. https://doi.org/10.1080/ 21670811.2014.927986

Lewis, S. C., \& Zamith, R. (2017). On the worlds of journalism. In P. J. Boczkowski \& C. W. Anderson (Eds.), Remaking the news: Essays on the future of journalism scholarship in the digital age (pp. 111-128). Cambridge, MA: MIT Press.

Lindén, C.-G. (2017). Algorithms for journalism: The future of news work. The Journal of Media Innovations, 4(1), 60-76. https://doi.org/10.5617/jmi.v4i1.2420

Marlow, J., Dabbish, L., \& Herbsleb, J. (2013). Impression formation in online peer production. In Proceedings of the 2013 conference on computer supported cooperative work (pp. 117-128). New York, NY: ACM. https://doi.org/10.1145/2441776.2441792

Örnebring, H., \& Möller, C. (2018). In the margins of journalism. Journalism Practice, 12(8), 1051-1060. https://doi.org/10.1080/17512786.2018.1497455

Sandberg, J., Holmström, J., Napier, N., \& Levén, P. (2015). Balancing diversity in innovation networks. European Journal of Innovation Management, 18(1), 44-69. https://doi.org/10.1108/EJIM-09-2013-0088

Singer, J. B. (2007). Contested autonomy. Journalism Studies, 8(1), 79-95. https://doi.org/10.1080/ 14616700601056866

Smit, G., de Haan, Y., \& Buijs, L. (2014). Working with or next to each other? Boundary crossing in the field of information visualisation. The Journal of Media Innovations, 1(2), 36-51. https://doi.org/10.5617/jmi. v1i2.875

Star, S. L., \& Griesemer, J. R. (1989). Institutional ecology, "translations" and boundary objects: Amateurs and professionals in Berkeley's museum of vertebrate zoology, 1907-39. Social Studies of Science, 19(3), 387-420. https://doi.org/10.1177/ 030631289019003001

Stark, D. (2009). The sense of dissonance: Accounts of worth in economic life. Princeton, NJ: Princeton University Press.

Tsay, J., Dabbish, L., \& Herbsleb, J. (2014). Influence of social and technical factors for evaluating contribution in GitHub. In Proceedings of the 36th international conference on software engineering ( $\mathrm{pp}$. 356-366). New York, NY: ACM. https://doi.org/10. $1145 / 2568225.2568315$

Usher, N. (2016). Interactive journalism: Hackers, data, and code. Champaign, IL: University of Illinois Press.

Usher, N. (2017). Venture-backed news startups and the field of journalism. Digital Journalism, 5(9), 1116-1133. https://doi.org/10.1080/21670811. 2016.1272064

Vendome, C., Linares-Vásquez, M., Bavota, G., di Penta, M., German, D., \& Poshyvanyk, D. (2015). License usage and changes: A large-scale study of java projects on GitHub. In Proceedings of the 2015 IEEE 23rd international conference on program comprehension ( $\mathrm{pp}$. 218-228). Piscataway, NJ: IEEE Press.

Vos, T. P., \& Craft, S. (2017). The discursive construction of journalistic transparency. Journalism Studies, 18(12), 1505-1522. https://doi.org/10.1080/ 1461670X.2015.1135754

Wahl-Jorgensen, K. (2017). A manifesto of failure for digital journalism. In P. J. Boczkowski \& C. W. Anderson (Eds.), Remaking the news: Essays on the future of journalism scholarship in the digital age (pp. 251-266). Cambridge, MA: MIT Press.

Weber, S. (2004). The success of open source. Cambridge: Harvard University Press.

Weber, M. S., \& Kosterich, A. (2018). Coding the news. Digital Journalism, 6(3), 310-329. https://doi.org/10. 1080/21670811.2017.1366865

Witt, L. (2006). Constructing a framework to enable an open source reinvention of journalism. First Monday, 11(6). Retrieved from https://firstmonday.org/ ojs/index.php/fm/article/view/1338/1258

Wu, S., Tandoc, E. C., \& Salmon, C. T. (2019). Journalism reconfigured. Journalism Studies, 20(10), 1440-1457. https://doi.org/10.1080/1461670X.2018.1521299

Zamith, R. (2019). Algorithms and journalism. In Oxford encyclopedia of journalism studies (1-21). Oxford: Oxford University Press. https://doi.org/10. 1093/acrefore/9780190228613.013.779

Zamith, R., Belair-Gagnon, V., \& Lewis, S. C. (2019). Constructing audience quantification: Social influences and the development of norms about audi- 
ence analytics and metrics. New Media \& Society. Advance online publication. https://doi.org/10.1177/ 1461444819881735

Zamith, R., \& Braun, J. A. (2019). Technology and journalism. In T. P. Vos, F. Hanusch, D. Dimitrakopoulou, M.
Geertsema-Sligh, \& A. Sehl (Eds.), International encyclopedia of journalism studies (pp. 1-8). Hoboken, NJ: Wiley. https://doi.org/10.1002/9781118841570. iejs0040

\section{About the Authors}

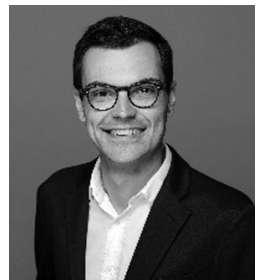

Mario Haim (PhD, University of Stavanger) is a Postdoctoral Fellow to the Department of Media and Social Sciences at the University of Stavanger, Norway. His research interests include algorithmic influences on journalism, such as within automated and data-driven journalism as well as through audience metrics, the use of news within algorithmically curated media environments, and the technological means of online health communication. He also has a keen interest in computational methods. More information can be found at https://www.haim.it

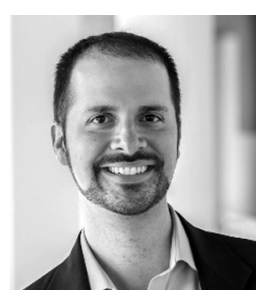

Rodrigo Zamith (PhD, University of Minnesota) is an Assistant Professor in the Journalism Department at the University of Massachusetts Amherst and a Faculty Associate at UMass' Computational Social Science Institute. His research interests lie at the intersection of journalism and technology, with a focus on the reconfiguration of journalism in a changing media environment and the development of digital research methods for social scientists. Zamith's most recent work can be found at https://www.rodrigozamith.com 